\title{
EXPANSIVENESS AND HYPERBOLICITY IN CONVEX BILLIARDS
}

\author{
MÁRIO BESSA, JOÃO LOPES DIAS, AND MARIA JOANA TORRES
}

\begin{abstract}
We say that a convex planar billiard table $B$ is $C^{2}$-stably expansive on a fixed open subset $U$ of the phase space if its billiard map $f_{B}$ is expansive on the maximal invariant set $\Lambda_{B, U}=$ $\cap_{n \in \mathbb{Z}} f_{B}^{n}(U)$, and this property holds under $C^{2}$-perturbations of the billiard table. In this note we prove for such billiards that the closure of the set of periodic points of $f_{B}$ in $\Lambda_{B, U}$ is uniformly hyperbolic. In addition we show that this property also holds for a generic choice among billiards which are expansive.
\end{abstract}

MSC 2010: 37D20, 37D50, 37C20.

Keywords: convex planar billiards, hyperbolic sets, expansiveness.

Original publication:

Regular and Chaotic Dynamics, 26 (2021), 756-762

DOI: $10.1134 / \mathrm{S} 1560354721060125$

\section{INTRODUCTION}

In a few words, a dynamical system is expansive if whenever two points stay near for forward and backward iterates, they actually coincide. This notion was first developed in the 1950's (see [25]) related to the fact that these systems exhibit sensitivity to the initial conditions. Uniform hyperbolicity implies expansivity as it was understood in the first half of the 1970's [8, 16]. In particular, Anosov maps (global hyperbolicity) and Axiom A maps (local hyperbolicity) are expansive.

It is well known that in certain classes of conservative dynamical systems, the robusteness of certain properties ensures some kind of hyperbolicity. Examples include expansiveness [20], ergodicity [24], transitivity [2], shadowing [4, 5, 6], weak shadowing [4] and topological stability [4]. Here we are interested in the class of expansive convex planar billiards for which there are few related results. We propose to answer the following questions. How does a robust expansiveness property lead to hyperbolicity? What distinguishes hyperbolicity from expansiveness, from a generic point of view?

Related to the questions above, we recall the works for geodesic flows of Ruggiero on the stability of expansiveness [20], and of the first author on the generic characterization of expansiveness and non-trivial hyperbolic basic sets [1].

Finally, it is worth observing that there are (degenerate) examples of non-hyperbolic fixed points for expansive area-preserving maps (cf. [4, Figure 1]). However those examples are not robust. Notice also that, contrary to what happens for geodesic flows, there is no global hyperbolicity for sufficiently smooth convex billiards. Hence, in the present setting we consider a local approach related to a fixed set $U$ in phase space. It is of course known that nontrivial hyperbolic sets can exist in a local setting for convex billiards (see for example [14]).

1.1. Statement of the results. As detailed in $\$ 2.1$, a $C^{2}$ billiard table $B$ generates a $C^{1}$ symplectomorphism $f_{B}$ called the billiard map. In the following, whenever there is no ambiguity, we refer to $B$ being $C^{2}$ and $f_{B}$ being $C^{1}$ indistinctly. 
Consider a convex billiard $B$ and the corresponding phase space $M$ with a metric $d$. Let $U$ be an open subset of $M$.

Definition 1.1. The billiard map $f_{B}: M \rightarrow M$ is called expansive on $\Lambda \subset U$ if there is $e>0$ (the expansive constant) such that given $x \in \Lambda$ and $y \in M$ if $d\left(f_{B}^{n}(x), f_{B}^{n}(y)\right) \leq e$ for all $n \in \mathbb{Z}$, then $x=y$ (cf. [22, Definition 7.3]). When $\Lambda=M$, we say that $f_{B}$ is expansive. Moreover, $B$ is $C^{2}$-stably expansive on $U$ if $f_{B}$ is expansive on the maximal invariant set

$$
\Lambda_{B, U}=\cap_{n \in \mathbb{Z}} f_{B}^{n}(U),
$$

and for any $\tilde{B}$ sufficiently $C^{2}$-close to $B$ the map $f_{\tilde{B}}$ is also expansive on $\Lambda_{\tilde{B}, U}$.

Our first result shows that the $C^{2}$-stably expansive topological property implies hyperbolicity. We denote by $\operatorname{Per}\left(f_{B}\right)$ the set of periodic points of $f_{B}$.

Theorem 1. Let $U \subset M$ be open. If $B$ is $C^{2}$-stably expansive on $U$, then $\overline{\operatorname{Per}\left(f_{B}\right)} \cap \Lambda_{B, U}$ is uniformly hyperbolic.

Remark 1.1. Under the conditions of the previous theorem, it follows from standard arguments in hyperbolic dynamics (see e.g. proof of [13, Theorem D]) that if the number of periodic points in $\Lambda_{B, U}$ is infinite, then $\overline{\operatorname{Per}\left(f_{B}\right)} \cap \Lambda_{B, U}$ is a non-trivial hyperbolic basic set.

The strategy of the proof of Theorem 1 relies on the combination of several results on billiard and area-preserving maps dynamics. We highlight in particular the following three recent results:

(1) The Mañé dichotomy (Theorem 2.2) is an abstract cocycle formulation. It decomposes closed orbits on $U$ in either hyperbolic ones or else in orbits that can be perturbed to parabolic.

(2) The Visscher's version of Franks' lemma for planar billiards (Theorem 2.1) permits the application of the abstract formulation of Mañés dichotomy to billiard maps.

(3) The Bunimovich and Grigo's technical theorem (Theorem 2.3) allows us to perturb the billiard table preserving the expansiveness hypothesis and simultaneously transforming a parabolic or elliptic point into a Moser stable elliptic point ${ }^{1}$. This is crucial in obtaining a contradiction to the expansiveness hypothesis.

We emphasize the restriction to the $C^{2}$-topology for convex billiard tables (i.e. to the $C^{1}$-topology for the billiard map) since the above results are proved only for this topology (see §3).

Consider the residual subset $\mathscr{R}$ of convex planar billiards given in [15, 27]. It corresponds to maps that verify the property that all closed orbits are hyperbolic or irrationally elliptic. KAM-type arguments applied to a sufficiently smooth billiard table $B$ satisfying the expansiveness property imply that there are no irrationally elliptic closed orbits. Therefore, a generic expansive map on $\Lambda_{B, U}$ has all its closed orbits of hyperbolic type. A much more demanding question is whether the closure of the hyperbolic closed orbits in $\Lambda_{B, U}$ is a uniformly hyperbolic set. An answer is given in Theorem 2 by showing that seemingly different concepts like expansiveness and hyperbolicity are equivalent from a generic point of view.

Theorem 2. Let $U \subset M$ be open. There exists a $C^{2}$-residual subset $\mathscr{R}$ of the set of $C^{2}$ convex billiard tables such that if $B \in \mathscr{R}$, then $f_{B}$ is expansive on $\overline{\operatorname{Per}\left(f_{B}\right)} \cap \Lambda_{B, U}$ if and only if $\overline{\operatorname{Per}\left(f_{B}\right)} \cap \Lambda_{B, U}$ is uniformly hyperbolic.

The proof is contained in $\S 4$. In $\S 2$ we present the basic definitions to understand the formal statements of the results.

\footnotetext{
${ }^{1} \mathrm{~A}$ periodic point is Moser stable if it is surrounded by toric invariant curves in arbitrarily small neighborhoods. Moreover, each of these toric $f_{B}$-invariant curves $\gamma$ is such that $\left.f_{B}\right|_{\gamma}$ is transitive, in the sense it contains a dense orbit.
} 


\section{Preliminaries}

2.1. Convex billiard tables and maps. A billiard table $B$ is a bounded, open and connected domain of $\mathbb{R}^{2}$ with boundary $\partial B$. The flow on its unit tangent bundle $S B$ generated by the motion of a free point-particle in $B$ with specular reflection at $\partial B$ (the angle of reflection equals the angle of incidence) is called the billiard flow. Let the phase space $M \subset S(\partial B)$ be the set of unit tangent vectors at each point in $\partial B$ that are pointing inside the billiard table $B$. The billiard map $f_{B}$ is the first return map on $M$ of the billiard flow (cf. [12]).

We are interested in convex billiard tables with a $C^{k}$-boundary $\partial B, k \geq 2$. This implies that $f_{B}$ is a $C^{k-1}$ diffeomorphism on $M$. It is convenient to use coordinates on $M$. By writing $\alpha: \mathbb{R} / l \mathbb{Z} \rightarrow \mathbb{R}^{2}$ as the arc-length parametrization of $\partial B$, where $l$ is the length of $\partial B$, each $x \in M$ can be simply written as $x=(s, \varphi)$. Here $s$ is the arc-length parameter of $\partial B$ of the base point of $x$, and $\varphi$ is the angle between $x$ and the positively oriented tangent to $\partial B$ at $\alpha(s)$ (the reflected angle). So,

$$
(s, \varphi) \in M=[0, l[/ \sim \times[0, \pi]
$$

where $s \sim s^{\prime}$ if $s=s^{\prime}$ mod $l$. Moreover, the derivative of $f_{B}$ (see [12]) is given by:

$$
D f_{B}(s, \varphi)=-\frac{1}{\cos \varphi_{1}}\left[\begin{array}{cc}
\tau K+\cos \varphi & \tau \\
\tau K K_{1}+K \cos \varphi_{1}+K_{1} \cos \varphi & \tau K_{1}+\cos \varphi_{1}
\end{array}\right],
$$

where $\left(s_{1}, \varphi_{1}\right)=f_{B}(s, \varphi), K$ and $K_{1}$ are the curvatures of $\partial B$ at $\alpha(s)$ and $\alpha\left(s_{1}\right)$, respectively, $\tau=\left\|\alpha(s)-\alpha\left(s_{1}\right)\right\|$ and $\|\cdot\|$ denotes the Euclidean norm on $\mathbb{R}^{2}$. The map $f_{B}$ preserves the measure $\mu$ given by $d \mu=\cos \varphi d \varphi d s$. Notice that $\operatorname{det} D f_{B}(s, \varphi)=\cos \varphi / \cos \varphi_{1}$. Therefore, for a periodic point $p=(s, \varphi)$ with period $\ell$, we have $\operatorname{det} D f_{B}^{\ell}(p)=1$.

Each billiard table $B$ is identified with its boundary $C^{k}$-curve $\partial B$, which in turn can be described up to reparametrization by a $C^{k}$-embedding $\alpha: S^{1} \rightarrow \partial B$, where $S^{1}=\mathbb{R} / \mathbb{Z}$. Recall that at each point $\alpha(s)$ there is a unit normal vector $n(s)$ to the curve, and $s \mapsto n(s)$ can be chosen to be $C^{1}$. Moreover, the dynamics associated to a billiard table is clearly preserved by isometries and homotheties of the plane. So, we will consider the space of convex billiard tables $\mathcal{B}$ as the set of embeddings modulo those transformations and reparametrizations.

Given $\varepsilon>0$ we say the $C^{2}$-embeddings $\alpha: S^{1} \rightarrow \mathbb{R}^{2}$ and $\beta: S^{1} \rightarrow \mathbb{R}^{2}$ are $\varepsilon$ - $C^{2}$-close if there is a $C^{2}$ map $\lambda: S^{1} \rightarrow \mathbb{R}^{2}$ such that

$$
\|\lambda\|_{C^{2}}<\varepsilon \text { and } \beta(s)=\alpha(s)+\lambda(s) n_{\alpha}(s), \quad s \in S^{1},
$$

where $n_{\alpha}(s)$ is the unit normal vector of the curve $\alpha\left(S^{1}\right)$ at $\alpha(s)$. Notice that the same is true if we consider instead a unit normal vector to $\beta$.

The above is used to define close billiard tables in $\mathcal{B}$. We say that two equivalent classes $[\alpha],[\beta] \in \mathcal{B}$ are $\varepsilon$ - $C^{2}$-close if there are representatives $\alpha \in[\alpha]$ and $\beta \in[\beta]$ which are $\varepsilon$ - $C^{2}$-close. As a result $\mathcal{B}$ is a Baire space [11].

\subsection{Hyperbolic, elliptic and parabolic period points.}

2.2.1. Uniform hyperbolicity and periodic points. Let $\Lambda \subset M$ be $f_{B}$-invariant. The $D f_{B}$-invariant splitting $E^{1} \oplus E^{2}$ of the tangent bundle $T \Lambda$ into 1-dimensional bundles $E^{1}$ and $E^{2}$ is said to be uniformly hyperbolic if there exists $\sigma \in(0,1)$ such that for any point $x \in \Lambda$ we have $\left\|\left.D f_{B}(x)\right|_{E_{x}^{1}}\right\| \leq \sigma$ and $\left\|\left.D f_{B}^{-1}(x)\right|_{E_{x}^{2}}\right\| \leq \sigma$. Equivalently, we can choose $\sigma=\frac{1}{2}$ and allow to iterate $m$ times to get $\left\|\left.D f_{B}^{m}(x)\right|_{E_{x}^{1}}\right\| \leq \frac{1}{2}$ and $\left\|\left.D f_{B}^{-m}(x)\right|_{E_{x}^{2}}\right\| \leq \frac{1}{2}$ for some $m \in \mathbb{N}$. In this last formulation we say that $f_{B}$ on $\Lambda$ is $m$-uniformly hyperbolic. The angle between $E^{1}$ and $E^{2}$, denoted by $\measuredangle\left(E^{1}, E^{2}\right)$, is bounded away from zero on uniform hyperbolic sets.

Given a periodic point $p$ of period $\ell$, the eigenvalues $\lambda, \lambda^{-1}$ of $D f_{B}^{\ell}(p)$ determine the type of local dynamics. The periodic point is 
- elliptic if $|\lambda|=1$ and $\lambda \in \mathbb{C} \backslash \mathbb{R}$,

- parabolic if $|\lambda|=1$ and $\lambda \in \mathbb{R}$,

- hyperbolic if $|\lambda| \neq 1$ and $\lambda \in \mathbb{R}$.

2.2.2. Periodic points of bounded fixed period. Notice that all periodic points of period two are in $\{\varphi=\pi / 2\}$, since the reflections are normal to $\partial B$.

If there are infinitely many periodic points of period two, then, by continuity of the map, they form a sequence with at least two accumulation points which are also periodic with period two. Write $x_{k} \in M$ for a subsequence converging to one of the accumulation points, say $x$. Notice also that $f_{B}\left(x_{k}\right) \rightarrow f_{B}(x)$ as $k \rightarrow+\infty$. So, for any choice of $e>0$ there is a large enough $k \in \mathbb{N}$ so that

$$
\max _{i=0,1} d\left(f_{B}^{i}\left(x_{k}\right), f_{B}^{i}(x)\right)<e .
$$

By the fact that they are period two orbits, there is no expansivity in this situation. Notice that the same reasoning holds for orbits of any other fixed period.

2.3. Main tools. The following theorem is a planar billiard version of the well-known Franks' lemma. We denote by $\mathrm{Sp}(2)$ the set of $2 \times 2$ symplectic real-valued matrices.

Theorem 2.1 (Visscher [21]). There exists a residual set $\mathcal{R}$ of $C^{k}$ convex billiards with $k \geq 2$ or $k=\infty$, such that for $B \in \mathcal{R}$, a periodic point $p$ of period $\ell \geq 3$, and a neighborhood $\mathcal{U}$ of $B$ in $C^{k}$ for the $C^{2}$-topology, there exists an open ball $\mathscr{B} \subset S p(2)$ around $D f_{B}^{\ell}(p)$ such that any element of $\mathscr{B}$ is realizable as $D f_{\tilde{B}}^{\ell}(p)$ for some $\tilde{B} \in \mathcal{U}$. Moreover, the perturbation can be supported in an arbitrarily small neighbourhood of three sequential points in the orbit of $p$ on the boundary of the table.

We point out that the perturbations whose existence is guaranteed by Theorem 2.1 are, of course, only $C^{2}$-small, despite the improvement in topology of the residual set. The above statement is actually a simple improvement of the result in [21] by noting that the residual set can be taken to be in the $C^{k}$ topology by the works of Stojanov and Petkov [17, 18, 19, 23]. Moreover, the perturbation is obtained through smooth bump functions and has therefore the same regularity of the original billiard.

We observe that the constraint on Theorem 2.1 about the size of the closed orbit $\gamma$ will be innocuous in the proof of Theorem 1.

The next result is in the spirit of the Mañé dichotomies on closed orbits: the dominated splitting is the sole obstruction to obtain trivial spectrum on closed orbits. The abstract general result for dissipative systems was obtained in [7, Corollary 2.19]. For Hamiltonian and geodesic flows see [3, section 3.1], [13, section 8] and [5]. In brief terms, assuming the non-dominance hypothesis, we can rotate the solutions along the closed orbits in order to mix different expansion rates. Since we have symmetry of the symplectic spectrum, these rates annihilate each other. Finally, as many rotations may be needed, the period of the closed orbits should be large enough.

Theorem 2.2 (Mañé dichotomy). Let $B \in \mathcal{R}$ where $\mathcal{R}$ was obtained in Theorem 2.1. For any $\eta>0$ there are $m \in \mathbb{N}$ and $n \geq 3$ such that for any periodic point $p$ of $f_{B}$ with period $\ell \geq n$, either

(i) $D f_{B}^{\ell}(p)$ admits an m-uniformly hyperbolic splitting ${ }^{2}$, or else

(ii) for any neighbourhood $V \subset M$ of the orbit of $p$, there exists a $C^{\infty}$ billiard table $\tilde{B} \eta$ - $C^{2}$-close to $B$ such that $f_{\tilde{B}}=f_{B}$ outside $V$ and $p$ is a parabolic periodic point of $f_{\tilde{B}}$.

We now recall a result due to Bunimovich and Grigo that will allow us to turn a parabolic or elliptic periodic orbit into Moser stable under a small $C^{5}$-perturbation.

\footnotetext{
${ }^{2}$ The original statement is $m$-uniformly dominated splitting. In the symplectic case can be specified as $m$-uniformly partial hyperbolic splitting, and in our 2-dimensional case, $m$-uniformly hyperbolic splitting.
} 
Theorem 2.3. Let $B$ be a $C^{5}$ convex billiard table and $p$ an irrationally elliptic periodic point. Then, there is a $C^{5}$-close convex billiard $\hat{B}$ for which the orbit of $p$ is preserved and it is Moser stable.

Proof. This is an immediate consequence of [9, Theorem 4].

Proposition 2.4. If $p \in U$ is an elliptic or parabolic periodic point of a $C^{2}$ convex billiard table $B$, then $B$ is not $C^{2}$-stably expansive on $U$.

Proof. By taking a $C^{2}$-close smooth billiard and then using Theorem 2.1, we obtain a $C^{2}$-perturbed smooth billiard with an irrationally elliptic periodic point. Then, Theorem 2.3 allows us to make $p$ Moser stable for a nearby billiard table $\hat{B}$. Given any $e>0$ we can always find an invariant curve $C$ surrounding $p$ such that $\max _{1 \leq n \leq \ell} \max _{y \in C} d\left(f_{\hat{B}}^{n}(y), f_{\hat{B}}^{n}(p)\right) \leq e$. This means that $\hat{B}$ is not expansive and $B$ is not $C^{2}$-stably expansive.

\section{Proof of Theorem I}

The hypothesis on the $C^{2}$-stability of the expansiveness property is incompatible with assertion (ii) of Theorem 2.2 and this will imply the proof of Theorem 1.

We start by showing that $\operatorname{Per}\left(f_{B}\right)$ restricted to $\Lambda_{B, U}$ is uniformly hyperbolic. Suppose, by contradiction, that $(s, \theta)=p \in U$ is an elliptic, parabolic or a hyperbolic periodic point with very weak hyperbolicity. Being elliptic or parabolic is immediately excluded by Proposition 2.4.

Let $\ell$ be the period of $p$ and let $n, m$ be given by Theorem 2.2 .

(1) $(2 \leq \ell<n)$ If there are a finite number of those hyperbolic periodic points, then, as a set, it is uniformly hyperbolic. If there are an infinite number of these hyperbolic periodic points, then by the arguments in $\$ 2.2 .2$ we get a contradiction with the fact that we have expansivity.

(2) $(\ell \geq n)$ Since the spectral objects depend continuously on $B$ we get that $D f_{B}(p)$ admits an $\hat{m}$-uniformly hyperbolic splitting (eventually $\hat{m}>m$ ). This follows because item (ii) of Theorem 2.2 is excluded since we are considering that $B$ is $C^{2}$-stably expansive on $U$ and we are also using Theorem 2.1 to perform the perturbations.

In conclusion, we obtain that $\operatorname{Per}\left(f_{B}\right)$ restricted to $\Lambda_{B, U}$ is a uniformly hyperbolic set. The hyperbolicity condition implies the continuity of the splitting in $\operatorname{Per}\left(f_{B}\right)$ [19, Proposition 6.4.4]. Then the splitting extends continuously to $\overline{\operatorname{Per}\left(f_{B}\right)}$ and the extension is also hyperbolic.

\section{Proof of Theorem 2}

Since it is well-known from classic hyperbolic dynamics theory that uniform hyperbolicity implies expansiveness, we focus on the direct implication of Theorem 2 . We begin by proving the following preliminary result.

Lemma 4.1. Let $B$ be a $C^{2}$ convex billiard. If $f_{B}$ has a non-hyperbolic periodic point $p$ of period $\ell$, then for all $\epsilon, v>0$ there exists $\hat{B}$ such that:

(1) $\hat{B}$ is $\epsilon$ - $C^{2}$-close to $B$,

(2) $f_{\hat{B}}$ has two hyperbolic periodic points $p_{1}$ and $p_{2}$ (not belonging to the same orbit) with equal period and

(3) $\max _{1 \leq n \leq \ell} d\left(f_{\hat{B}}^{n}\left(p_{1}\right), f_{\hat{B}}^{n}\left(p_{2}\right)\right)<v$.

Proof. By Lazutkin's theorem [15] (see also [27]) $C^{\infty}$ generically, every periodic point is either hyperbolic or elliptic with irrational rotation number. Hence, using also Theorem 2.3, B can be perturbed in order to obtain an elliptic periodic point of period $\ell$ arbitrarily near $p$ which is Moser stable. A $C^{2}$-perturbation as in [14] guarantees also the existence of transverse homoclinic points arbitrarily close to the elliptic periodic point $(c f$. $[26,10]$ since convex billiard maps are area-preserving 
twist maps). Hence, there are invariant sets (Smale horseshoes) which are topologically equivalent to the full shift in every neighborhood of the elliptic point. In particular we can find hyperbolic periodic points fulfilling (2). Both hyperbolic periodic points and their iterates are inside a union of 'trapping regions' (each surrounded by an invariant curve due to Moser stability) of small enough diameters $<v$ and so accomplishing (3).

Let $U \subset M$ be open. Consider the subset $\mathscr{F}^{2}(U)$, the so-called Mané star systems in $U$, consisting of convex billiards $B$ such that all periodic orbits in $\operatorname{Per}\left(f_{\hat{B}}\right) \cap \Lambda_{\hat{B}, U}$ are hyperbolic for any convex billiard $\hat{B} C^{2}$-sufficiently close to $B$.

Proposition 4.2. Let $U \subset M$ be open. There exists a $C^{2}$-residual subset $\mathcal{R}$ of the set of $C^{2}$ convex billiard tables, such that if $B \in \mathscr{F}^{2}(U) \cap \mathcal{R}$, then $\overline{\operatorname{Per}\left(f_{B}\right)} \cap \Lambda_{B, U}$ is uniformly hyperbolic.

Proof. From [15] we know that in a residual set of convex billiards there is a finite number of periodic points of period less than a given $n$. We intersect this residual with the one in Theorem 2.1 to get $\mathcal{R}$. Since $B \in \mathscr{F}^{2}(U)$ we have that item (ii) of Theorem 2.2 is excluded. Hence, the set $P_{1}$ of periodic points with large period admits an m-uniformly hyperbolic splitting. On the other hand, as the set $P_{2}$ of periodic points in $U$ with small period is finite, it also admits an $m_{2}$-uniformly hyperbolic splitting.

Lemma 4.3. Let $U \subset M$ be open. There exists a residual subset $\mathscr{R}$ of $C^{2}$ convex billiards, such that for any $B \in \mathscr{R} \backslash \mathscr{F}^{2}(U), f_{B}$ has two sequences of periodic points $\left\{p_{n}\right\}_{n \in \mathbb{N}}$ and $\left\{q_{n}\right\}_{n \in \mathbb{N}}$ in $U$ such that for each $n, p_{n}$ and $q_{n}$ have distinct orbits and

$$
\lim _{n \rightarrow+\infty} \sup _{k \in \mathbb{Z}} d\left(f_{B}^{k}\left(p_{n}\right), f_{B}^{k}\left(q_{n}\right)\right)=0 .
$$

Proof. For each $n \in \mathbb{N}$, we denote by $\mathscr{N}_{n}$ the subset of convex billiard tables such that any $B \in \mathscr{N}_{n}$ has a $C^{2}$-neighborhood $\mathcal{U}$ with the following $C^{2}$-open property: for every $\hat{B} \in \mathcal{U}$, there are hyperbolic periodic points $p_{n}, q_{n}$ of $f_{\hat{B}}$ having distinct orbits such that

$$
\sup _{k \in \mathbb{Z}} d\left(f_{\hat{B}}^{k}\left(p_{n}\right), f_{\hat{B}}^{k}\left(q_{n}\right)\right)<\frac{1}{n} .
$$

Let $\mathscr{O}_{n}$ be the complement of the $C^{2}$-closure of $\mathscr{N}_{n}$ in the set of $C^{2}$ convex billiards. Clearly, $\mathscr{N}_{n} \cup \mathscr{O}_{n}$ is $C^{2}$-open and $C^{2}$-dense in the convex billiard tables set. We define the $C^{2}$-residual subset in the statement of the lemma by:

$$
\mathscr{R}=\bigcap_{n \in \mathbb{N}}\left(\mathscr{O}_{n} \cup \mathscr{N}_{n}\right)
$$

If $B \in \mathscr{R} \backslash \mathscr{F}^{2}(U)$, then there is a sequence of convex billiard tables $B_{j}$ converging to $B$ in the $C^{2}$-sense and a sequence of non-hyperbolic periodic points $\tilde{p}_{j}$ of $f_{B_{j}}$ in $U$. Then, for any $n \in \mathbb{N}$, by Lemma 4.1, we have that $B \in \mathscr{N}_{n}$, proving the claim.

From Lemma 4.3, we know that if $B$ belongs to the residual subset $\mathscr{R} \cap \mathcal{R}$ and it satisfies the expansiveness property on $\overline{\operatorname{Per}\left(f_{B}\right)} \cap \Lambda_{B, U}$, then it must be in $\mathscr{F}^{2}(U)$. By Proposition 4.2, $\overline{\operatorname{Per}\left(f_{B}\right)} \cap \Lambda_{B, U}$ is uniformly hyperbolic. This completes the proof of Theorem 2.

\section{ACKNOWLEDGEMENTS}

The authors were partially funded by the project "New trends in Lyapunov exponents" PTDC/MATPUR/29126/2017 financed by Fundação para a Ciência e a Tecnologia, Portugal. MB would also like to thank CMUP for providing the necessary conditions in which this work was developed. JLD was also partially funded by the Project CEMAPRE - UID/MULTI/00491/2019 financed by Fundação para a Ciência e a Tecnologia. MJT was also partially financed by national funds 
through Fundação para a Ciência e a Tecnologia within the projects UIDB/00013/2020 and UIDP/00013/2020.

\section{REFERENCES}

[1] M. Bessa, A Note on Expansiveness and Hyperbolicity for Generic Geodesic Flows, Math Phys Anal Geom (2018) 21: 14.

[2] M. Bessa, J. Rocha, On $C^{1}$-robust transitivity of volume-preserving flows, J. Diff. Eq. 245 (2008), 3127-3143.

[3] M. Bessa, J. Rocha and M. J. Torres, Hyperbolicity and stability for Hamiltonian flows, J. Diff. Eq. 254 (2013), 309-322.

[4] M. Bessa, J. Rocha and M. J. Torres, Shades of hyperbolicity for Hamiltonians, Nonlinearity, 26 (10) (2013), 28512873.

[5] M. Bessa, J. Lopes Dias and M. J. Torres, On shadowing and hyperbolicity for geodesic flows on surfaces, Nonlinear Analysis: Theory, Methods \& Applications 55, (2017), 250-263.

[6] M. Bessa, J. Lopes Dias and M. J. Torres, Hyperbolicity through stable shadowing for generic geodesic flows, Physica D 406 (2020), 132423.

[7] C. Bonatti, N. Gourmelon and T. Vivier, Perturbations of the derivative along periodic orbits, Ergod. Th. \& Dynam. Sys. 26 (2006), 1307-1337.

[8] R. Bowen and P. Walters. Expansive one-parameter flows. J. Diff. Equat. 12 (1972), 180-193.

[9] L. Bunimovich and A. Grigo Focusing components in typical chaotic billiards should be absolutely focusing, Commun. Math. Phys. 293 (2010), 127-43.

[10] P. Le Calvez, Propriétés Dynamiques des Difféomorphismes de l'Anneau et du Tore, Astérisque 204, 1991.

[11] M.J.D. Carneiro, S.O. Kamphorst and S. Pinto-de-Carvalho, Periodic orbits of generic oval billiards, Nonlinearity 20 (2007), 2453-2462.

[12] N. Chernov and R. Markarian, Chaotic billiards, Math. Surv. and Mono. 127, AMS, 2006.

[13] G. Contreras, Geodesic flows with positive topological entropy, twist maps and hyperbolicity, Annals Math. 172 (2010), 761-808.

[14] V. Donnay Creating transverse homoclinic connections in planar billiards J. Math. Sci. 128 (2005), 2747-53.

[15] V.F. Lazutkin, Convex billiard and eigenfunctions of the Laplace operator, Leningrad Univ. Press, 1981.

[16] R. Mãné, Expansive diffeomorphisms, Lecture Notes in Mathematics, Springer, 468 (1975), 162-174.

[17] V. Petkov and L. Stojanov, Periods of multiple reflecting geodesics and inverse spectral results, Amer. J. Math. 109 (1987), 619-668.

[18] V. Petkov and L. Stojanov, Spectrum of the Poincaré map for periodic reflecting rays in generic domains, Math. Z. 194 (1987), 505-518.

[19] V. Petkov and L. Stojanov, On the number of periodic reflecting rays in generic domains, Ergodic Theory Dynam. Systems 8 (1988), 81-91.

[20] R. Ruggiero, Persistently expansive geodesic flows. Comm. Math. Phys. 140 (1991), 203-215.

[21] D. Visscher, A Franks' lemma for convex planar billiards, Dynamical Systems 30 (2015), 333-340.

[22] M. Shub, Global Stability of Dynamical Systems, Springer-Verlag, 1987.

[23] L. Stojanov, Generic properties of periodic reflecting rays, Ergodic Theory Dynam. Systems 7 (1987), 597-609.

[24] A. Tahzibi, Robust transitivity and almost robust ergodicity, Ergodic Theory Dynam. Systems 24 (2004), 1261-1269.

[25] R. F. Williams, A note on unstable homeomorphisms, Proc. Amer. Math. Soc., 6 (1955), 308-309.

[26] E. Zehnder, Homoclinic points near elliptic fixed points, Comm. Pure Appl. Math. 26 (1973), 131-182.

[27] P. Zhang, Convex billiards on convex spheres, Ann. I.H.Poincaré-AN, 34 (2017), 793-816.

Departamento de Matemática, Universidade da Beira Interior, Rua Marquês d’Ávila e Bolama, 620i-ooi Covilhã, Portugal.

Email address: bessa@ubi.pt

Departamento de Matemática, CeMAPRE and REM, ISEG, Universidade de Lisboa, Rua do Quelhas 6, I200-78I Lisboa, Portugal

Email address: jldias@iseg.ulisboa.pt

CMAT and Departamento de Matemática, Universidade do Minho, Campus de Gualtar, 4700-057 Braga, Portugal Email address: jtorres@math.uminho.pt 